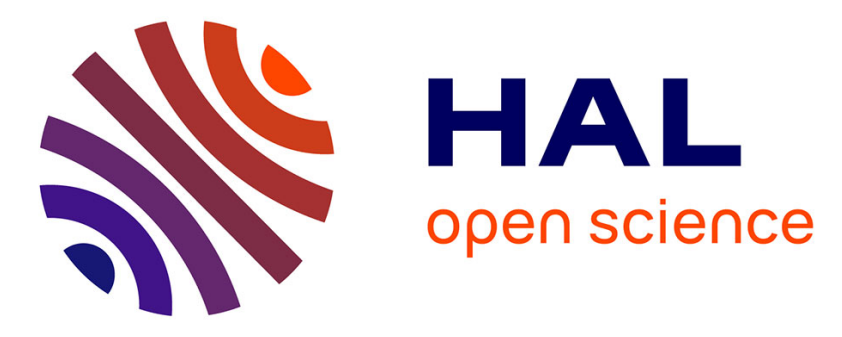

\title{
Patient-specific computational models: Tools for improving the efficiency of Medical Compression Stockings
}

Laura Dubuis, Pierre-Yves Rohan, Stéphane Avril, Pierre Badel, Johan Debayle

\section{To cite this version:}

Laura Dubuis, Pierre-Yves Rohan, Stéphane Avril, Pierre Badel, Johan Debayle. Patient-specific computational models: Tools for improving the efficiency of Medical Compression Stockings. International Conference on Medical Image Computing and Computer Assisted Intervention (MICCAI), Oct 2012, Nice, France. hal-00761911

\section{HAL Id: hal-00761911 https://hal.science/hal-00761911}

Submitted on 7 Jan 2013

HAL is a multi-disciplinary open access archive for the deposit and dissemination of scientific research documents, whether they are published or not. The documents may come from teaching and research institutions in France or abroad, or from public or private research centers.
L'archive ouverte pluridisciplinaire HAL, est destinée au dépôt et à la diffusion de documents scientifiques de niveau recherche, publiés ou non, émanant des établissements d'enseignement et de recherche français ou étrangers, des laboratoires publics ou privés. 


\title{
Patient-specific computational models: Tools for improving the efficiency of Medical Compression Stockings
}

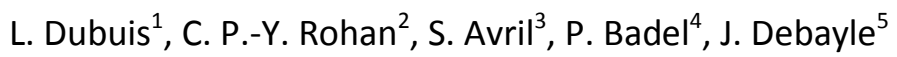 \\ PhD, Cardiovascular Research Unit, Chris Barnard Division of Cardiothoracic Surgery, Universi- \\ ty of Cape Town, South Africa. \\ ${ }^{2}$ PhD student, École Nationale Supérieure des Mines, CIS, CNRS: UMR 5146, LCG, F-42023 \\ Saint-Étienne, France. \\ ${ }^{3}$ Professor, École Nationale Supérieure des Mines, CIS, CNRS: UMR 5146, LCG, F-42023 Saint- \\ Étienne, France. \\ ${ }^{4}$ Associate Professor, École Nationale Supérieure des Mines, CIS, CNRS: UMR 5146, LCG, F- \\ 42023 Saint-Étienne, France. \\ ${ }^{5}$ Associate Professor, École Nationale Supérieure des Mines, CIS, CNRS: FRE 3312, LPMG, F- \\ 42023 Saint-Étienne, France.
}

\begin{abstract}
Compression therapy is used in the management and the treatment of various forms of venous insufficiency ranging from the relief of heavy and achy legs to the treatment of more severe forms such as acute venous ulceration. However, the pressure needed to achieve clinical benefit is a matter of debate. The purpose of this study was to examine the transmission of pressure within the soft tissues to improve current understanding of the mechanism of action of Medical Compression Stockings (MCS). Three-dimensional patient-specific finite element models were developed for six subjects. The geometry was obtained from CT-scans. Because experimental data on the mechanical properties of healthy adipose tissues and passive muscle are scarce in literature, an inverse method was setup to identify the constitutive properties of the said anatomical elements. This constitutes the original contribution of this work. The main outcome of this study is that the mean pressure applied by the MCS onto the skin is of the same order of magnitude as that applied by the compressed tissues onto the wall of the main deep veins, thereby suggesting that the mean pressure applied can be used as an indicator of the efficiency. Likewise, the maximal hydrostatic pressure in fat can be used to estimate the comfort.
\end{abstract}




\section{INTRODUCTION}

Compression therapy is a highly effective modality for treating venous disorders of the lower leg. It is traditionally employed to achieve a variety of therapeutic goals which mainly fall under three categories: prevention of veinrelated diseases such as varicose veins and deep vein thrombosis, the relief of symptoms associated with various forms of venous insufficiency and the treatment of venous-related diseases such as the treatment of active venous ulcerations [1].

It has been observed in practice however that the response of the calf to external compression is highly variable[2][3]. Conflicting results have also been reported in the case of injection sclerotherapy for varicose veins combined with external compression[4][5]. This highlights the current lack of knowledge regarding exact nature of the mechanical and biological responses of the leg to external compression.

One explanation brought forward by many authors is that the pressure performance of the MCS, which condition the effectiveness of the therapy, is particularly dependent on the morphology of the leg of each individual [6]. Much work has been done in that regards in order to evaluate the contact pressure performance of elastic stockings and to develop new tools to help manufacturers improve the medical functions and comfort of compression hosieries [7][8].

Yet, very little is known on the biomechanical response of the leg to this interface pressure and the implications for the venous return. The resulting redistribution of pressure is known to be non-uniform and is likely to be of paramount importance in the evaluation of MCS and to better comprehend the mechanisms by which it achieves its medical functions.

The purpose of this study was to simulate the three-dimensional distribution of pressure in the calf resulting from external compression on a significant number of patients. In order to address the issue of identifying the elastic properties of the soft-tissues, a Finite-Element Model Updating-based approach was developed and applied to six subjects. We have recently reported the results of the developed methodology on three subjects in [9]. 


\section{METHOD}

\subsection{Leg imaging protocol}

The right leg of six healthy volunteers was imaged using CT-scan (Computed Tomography scans) according to the following procedure. First, informed consent was obtained from all the volunteers according to a protocol approved by the local institutional ethics committee. A medical examination was then carried out to make sure that none of them suffered from venous insufficiency. Their leg perimeters were measured to prescribe the correct MCS in agreement with the manufacturer's recommendations. Finally, a CTscan was done on one of each subject leg with and without MCS. The sizes of the images were $512 * 512 * 376$ voxels and the resolution was $0.93 * 0.93 * 1$ $\mathrm{mm}^{3}$. The principal characteristics of each subject is summarised in Table 1 below.

Table 1. Subject characteristics

\begin{tabular}{|c|c|c|c|c|c|c|}
\hline Subjects & 1 & 2 & 3 & 4 & 5 & 6 \\
\hline Age (years old) & 42 & 25 & 30 & 35 & 35 & 25 \\
\hline Weight (kg) & 60 & 58 & 73 & 55 & 60 & 58 \\
\hline Height (m) & 1.6 & 1.7 & 1.8 & 1.7 & 1.7 & 1.6 \\
\hline Sex & Female & Female & Male & Female & Female & Female \\
\hline
\end{tabular}

\subsection{Definition of the model geometry}

\section{Segmentation step}

The three-dimensional CT-scan images were segmented into three main regions:

1. superficial soft tissues, composed principally of adipose tissues, skin and some veins, 
2. deep soft tissues, composed principally of muscles, tendons and blood vessels,

3. hard tissues, consisting of the two bones (the tibia and the fibula).

The segmentation was performed using the image processing program Image $\mathrm{J}^{\odot}$. A three-dimensional visualisation of the segmentation for one subject is shown in Fig. 1 below.

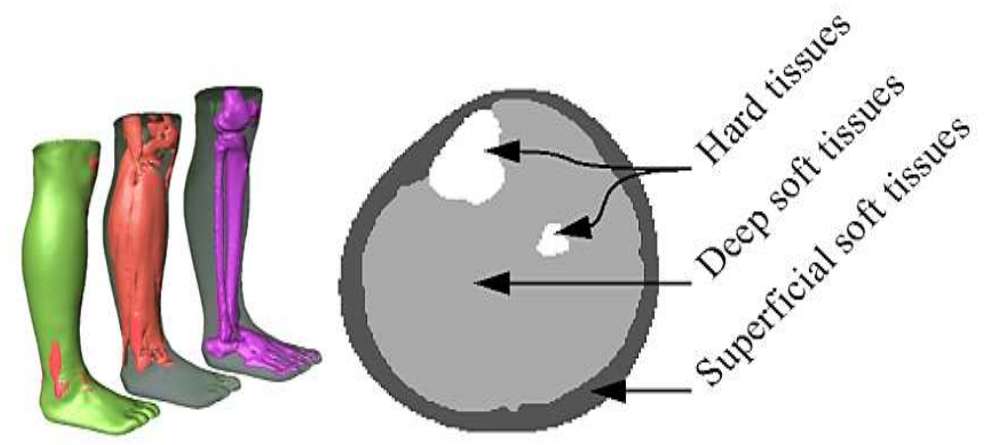

Fig. 1. Segmentation results: three-dimensional visualisation (left) and a cross section with the 3 main regions (right)

\section{Image warping}

Both the images of the legs with and without MCS need to be in the same reference so that the inverse method can be applied. This is achieved by performing a rigid transformation of the two geometries. The correct transformation is calculated from the formula (1) below which computes the smallest distance in the least-squares sense to superimpose the hard tissues of both legs:

$$
[\widehat{R}, \widehat{T}]=\arg _{(R, T)} \min \sum_{i}^{N}\left\|Q_{i}-R P_{i}-T\right\|
$$

where $R$ and $T$ are the optimal rotation and translation respectively to superimpose the two images, $N$ is the pixel number assigned to the bones in the images, $Q_{i}$ and $P_{i}$ are the coordinates of the bones in the leg without and with MCS, respectively. Only the two bones were used to perform image warping because it is assumed that they are not deformed under external compression. 


\subsection{Finite Element (FE) model}

\section{Meshing}

The segmented images were meshed using $\mathrm{AVIZO}^{\odot}$ with linear tetrahedral elements (approximately 500,000 elements and 60,000 nodes for each model).

\section{Boundary conditions}

The tibia and fibula are considered as infinitely rigid materials with respect to the elastic properties of soft tissues, and hence were fixed in this model.

The interface pressure delivered the MCS on the skin was computed from Laplace's law given in equation (2) below:

$$
P=\operatorname{Stiff} \frac{\varepsilon}{R c}
$$

where Stiff is the stiffness of the sock textile (estimated from a previous study [10]), $R c$ the curvature radius of the leg with MCS (to have the pressure in the final state) in the horizontal plane and $\varepsilon$ is the strain of the sock in the horizontal plane. The latter was derived knowing leg and sock perimeters from the CT-scans.

\section{Constitutive equation}

Both soft tissues were assumed to be homogeneous, isotropic, quasiincompressible and governed by a Neo-Hookean strain energy function, $\phi$ defined in equation (3) as follows:

$$
\varphi=c_{10}\left(\overline{I_{1}}-3\right)+\frac{\kappa}{2}(J-1)^{2}
$$

where $c_{10}$ and $\kappa$ are the Neo-Hookean parameters driving the constitutive equation, $\overline{I_{1}}=\operatorname{Tr}\left(\overline{\boldsymbol{F}} . \overline{\boldsymbol{F}}^{t}\right)$ is the first deviatoric strain invariant and $J=\operatorname{det}(\boldsymbol{F})$ is the volume ratio. Due to incompressibility, the $\mathrm{k}$ parameter was fixed at $1 \mathrm{MPa}$ for both soft tissues (as estimated by the volume change between the two configurations i.e. with and without external compression) and the parameter $c_{10}$ was identified by an inverse method. 


\subsection{Identification}

\section{Inverse method}

Finite-Element Model Updating is used for the identification process. The principle is outlined in the diagram given in Fig. 2 below. First, the patientspecific FE model is created from the initial imaging data (CT-scans of legs without external compression). The result of the simulation is then compared to the final experimental data (CT-scans of legs with MCS) based on a cost function, detailed below, which estimates the difference between the target leg contour (experimental images of leg under external compression) and the simulated leg contour. If the match between the two contours is not satisfactory, new constitutive parameters are calculated by an optimisation algorithm and the process is reiterated. When the cost function's minimum is reached, the constitutive parameters are considered to be identified. The optimisation algorithm employed here is the Nelder Mead algorithm implemented in Matlab ${ }^{\circledR}$ as the fminsearch function. Also, the starting value for the $\mathrm{c}_{10}$ parameter was $3 \mathrm{kPa}$.

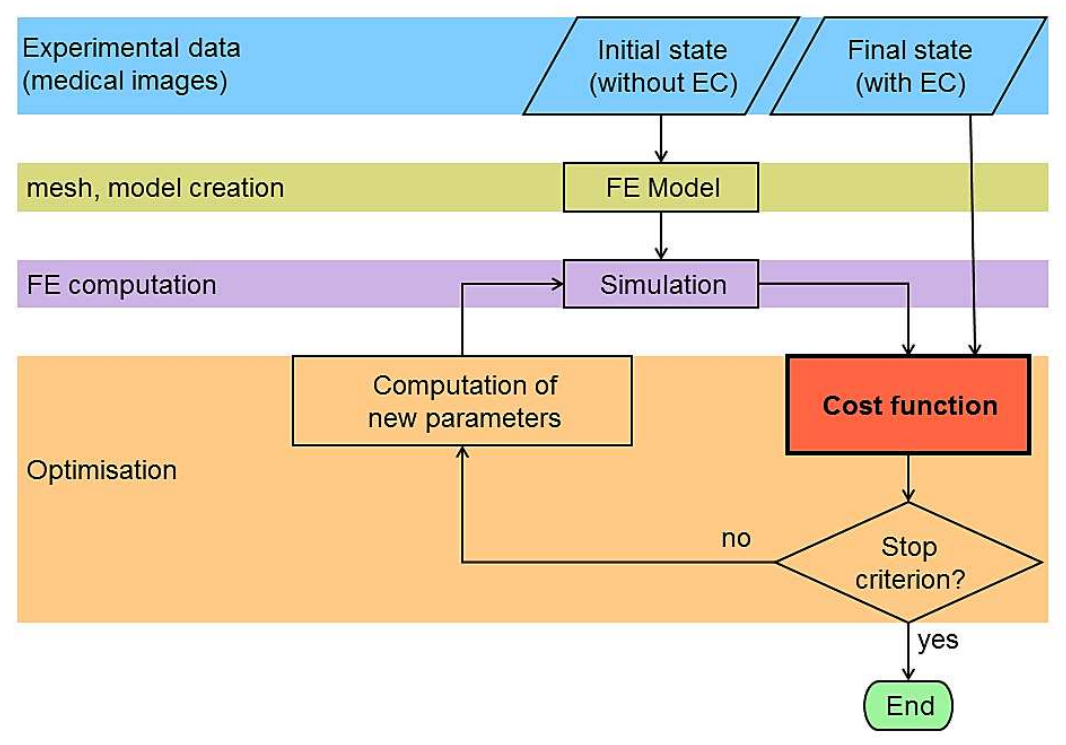

Fig. 2. Inverse Method 


\section{Cost function}

The cost function evaluates the average distance between the target leg contour and simulated deformed leg contour for five consecutive slices taken at the calf according to the formula (4) given below:

$$
\begin{aligned}
& \sum_{z=h 1}^{h 2} \sum_{\theta}\left(\left[\frac{r_{\text {simul }}(z, \theta)-r_{\text {target }}(z, \theta)}{r_{\text {target }}(z, \theta)}\right]_{\text {ext. }}^{2}-\left[\frac{r_{\text {simul }}(z, \theta)-r_{\text {target }}(z, \theta)}{r_{\text {target }}(z, \theta)}\right]_{\text {int. }}^{2}\right) \\
& C= \\
& \sum_{z=h 1}^{h 2} \sum_{\theta}\left(\left[\frac{r_{\text {initial }}(z, \theta)-r_{\text {target }}(z, \theta)}{r_{\text {target }}(z, \theta)}\right]_{\text {ext. }}^{2}-\left[\frac{r_{\text {initial }}(z, \theta)-r_{\text {target }}(z, \theta)}{r_{\text {target }}(z, \theta)}\right]_{\text {int. }}^{2}\right)
\end{aligned}
$$

where $r_{\text {simul }(z, \vartheta)}$ and $r_{\text {target }(z, \vartheta)}$ in the numerator are, respectively, the simulation and the target radius at the $\theta$ angle of the contours (Fig. 3b), for the outer (exterior) and inner (interior) contours of the superficial soft tissues (Fig. 3c) at the height $z$ of the leg; $h 1$ and $h 2$ are the boundary heights for the identification (Fig. 3a). In the denominator, $r_{\text {initial }(z, \vartheta)}$ represents the simulation contour at the beginning of the identification process.

The cost function takes values between 1 (at the first iteration of the Identification step) and 0 (when the simulated contours match the target experimental contours). The five slices covered a height of $3 \mathrm{~cm}$.
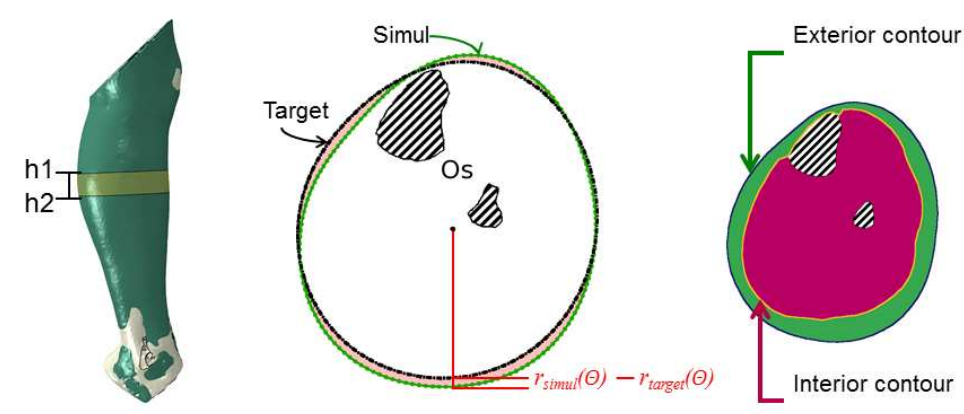

Fig. 3. Cost function. Minimisation of the difference of the target and simulation radius for all the contour of the leg (b) (minimisation of the red area on the scheme), for five slices between the heights $h_{1}$ and $h_{2}(a)$, and for the interior and exterior contours (c). 


\section{$3 \quad$ RESULTS}

\subsection{Identification results}

The average $c_{10}$ value identified for the deep soft tissue and for the six subjects was $3.25 \pm 0.93 \mathrm{kPa}$. The values of the cost function corresponding to the optimised constitutive parameter are given for each subject in the table 2 below.

Table 2. Values of the cost function at the end of the identification step for each subject

\begin{tabular}{|l|l|l|l|l|l|l|}
\hline Subjects & 1 & 2 & 3 & 4 & 5 & 6 \\
\hline Cost Function C & 0.85 & 0.73 & 0.44 & 0.19 & 0.21 & 0.44 \\
\hline
\end{tabular}

\subsection{Interface pressure distribution}

The interface pressure distribution for the six subjects, calculated from Laplace Law is illustrated in Fig. 4 below. It can be observed that the order magnitude of the applied pressures is highly non-uniform across the height of the leg. This highlights the inter-subject interface pressure variations and the need for patient-specific treatment of the simulation of the treatment.

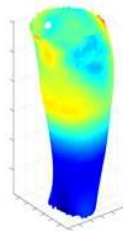

(a) Subject 1

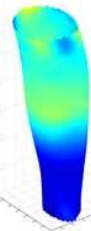

(b) Subject 2

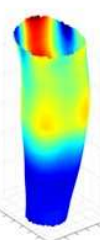

(c) Subject 3

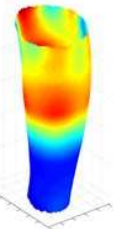

(d) Subject 4

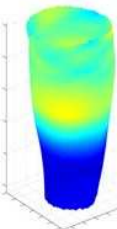

(e) Subject 5

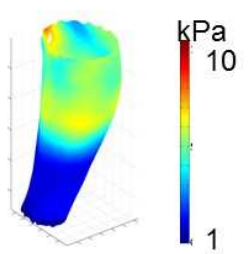

(f) Subject 6

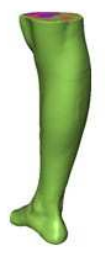

Fig. 4. Magnitude of the interface pressure distribution for each of the six subjects. A threedimensional rendering of the geometric model oriented in the same direction as six pressure maps is given in the Right Hand Side (R.H.S.) of the figure. 


\subsection{Pressure distribution}

The elastic properties of the soft tissues identified were used in the FEA to determine the pressure distribution in the calf when external compression is applied. The results for the hydrostatic pressure are shown for all the six subjects in Fig. 5 below. Hydrostatic pressure was chosen for the analysis because it does not depend on the coordinate system and can be used to predict local fluid flows. Although the appropriate MCS was used for each subject to ensure that comparable interface pressure was delivered from one subject to another, large inter-individual variability of the pressure field was observed in the results as illustrated in Fig. 5 below:

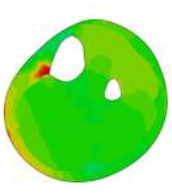

(a) Subject 1

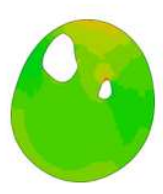

(b) Subject 2

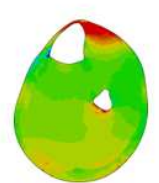

(c) Subject 3

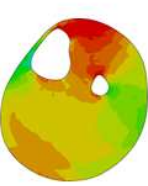

(d) Subject 4

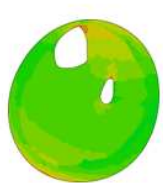

(e) Subject 5

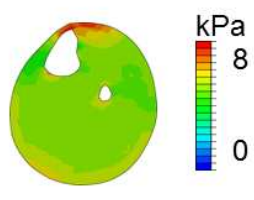

(f) Subject 6

Fig. 5. Hydrostatic Pressure field for six subjects at mid-calf in the leg cross section

Results also show that the pressure transmitted to the deep vein location is of the order of the average interface pressure delivered by the compression garment for most of the subjects. We can infer from this that the pressure delivered on the skin can be used as an indicator of the pressure effectively transmitted to the deep veins, and therefore of the efficiency of the MCS. The position of the main deep veins of the calf where the pressure transmitted was measured is illustrated in Fig. 6 below. 


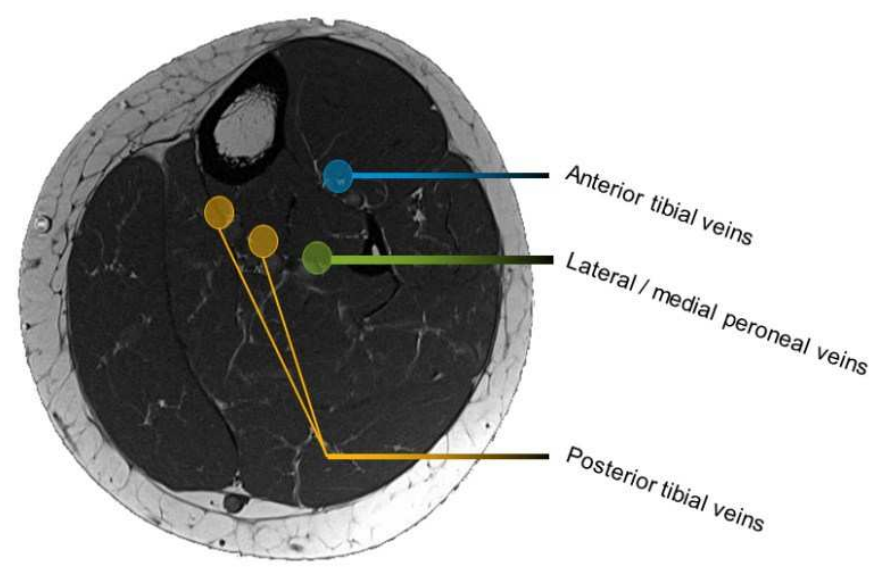

Fig. 6. Position in the calf of the major deep veins. The hydrostatic pressure predicted by the developed model was extracted in these locations to have an estimate of the pressure transmitted to the deep veins.

Moreover, due to the presence of pain receptors in the adipose tissue, it seems reasonable to think that the maximum hydrostatic pressure has a major contribution in the comfort sensed by patients wearing MCS. Our computational results (not shown here) suggest that there exists a relationship between the thickness of the adipose tissue and the maximum hydrostatic pressure. A direct clinical application of this is that thickness of the adipose tissue can be used as a gross evaluation of the comfort of the MCS.

\section{DISCUSSION}

\subsection{Interface pressure prediction using Laplace law}

In the present study, Laplace law has been used to compute the pressure exerted by the MCS on the surface of the skin and thus to define the natural loading conditions of the numerical model. The theoretical pressures derived from Laplace law have been shown to provide a good estimate of the real interface pressures measured using pressure transducers [7].

However, Laplace law is valid only insofar as solid non-deformable bodies are concerned. In the case of deformable bodies, as is the case here, it is crucial to take into account the mechanical interaction between the leg and the 
sock which results in variations of both the local radius of curvature of the limb and the local tension of the fabric. This particular point has been accounted for in the model by computing the surface pressure from the deformed geometry i.e. the CT-scans of the leg under compression.

Another approach consists in integrating the sock in the model and simulating the application of the latter on the leg [8] [11]. The simulation of the application of the sock has been developed in two-dimensions and results show that the distribution of pressure fit the one predicted by Laplace law.

In three-dimension, Laplace law was preferred to reduce the complexity of the problem, all the more so that Finite Element Updating requires a large number of simulations to determine the optimal material parameters.

\subsection{Choice of material behaviour law for the soft tissues}

The superficial and deep soft tissues constituting the model (i.e. the adipose tissue and muscle respectively) are defined as homogeneous, isotropic, compressible and hyperplastic materials.

The first assumption (homogeneity) stems from the fact that we are interested only in the deformation of the fat and muscle contours respectively because they are the only point of comparison between the predictions of the simulation and the experimental results. It follows that the local heterogeneities in the superficial and deep soft tissues are of no interest presently and are not considered in our model.

The second assumption originates chiefly from the experimental conditions: (i) the experimental protocol has been developed to evaluate the passive response of the muscle only and (ii) the leg is loaded in compression, essentially in a horizontal plane. Under these conditions, the muscle fibres have but a minor contribution in the response of the leg to external compression and have been considered as isotropic for the sake of simplicity. A more complex material behaviour - such as transverse isotropic for example would have been more realistic, but the benefits would not have outweighed the high cost of identifying the optimised material parameter set. 
Moreover, due to their high water content soft biological tissues can be approximated as nearly incompressible materials [12][13].

Finally, soft tissues are known to be highly non-linear materials exhibiting finite deformations. Under elastic compression, it has been observed that the deformation predicted by the model can be as high as $10 \%$ especially in calf region, as the soft tissues readjust and deform until the internal and exterior forces are balanced. A non-linear elastic model was therefore chosen to adequately reproduce experiments. The Neo-Hookean hyper-elastic material behaviour law was preferred because of the small number of parameters which simplify the identification process but yet is still a fully non-linear material. For small deformations, the Neo-Hookean model also reduces to the linear material model, which makes comparison with values from the literature easier.

\subsection{Identification results}

This result is in the same order of magnitude as that reported by [11] for in vivo indentation of pig muscle $(4.25 \mathrm{kPa})$. For the superficial soft tissue, the average identified value was $8.17 \pm 7.22 \mathrm{kPa}$, which is also close to the results reported in the literature by [12] for the buttock fat (11.7 kPa).

\subsection{Cost function}

The cost function presented above provides a measure of the gap between the experimental contour of the leg under compression and the contour predicted by the model under loading.

Although the uniqueness of the solution cannot be guaranteed, it has been tested for different algorithms and for different sets of initial parameters and the optimised parameter set was always the same (not show here).

The cost function has been defined for a slice of the leg taken in the calf instead of the whole leg to make sure that the assumption of homogeneity remains valid. Indeed, the constitutions of the soft tissues vary across the height of the leg. In the ankle, the presence of the tendons in the deep soft tissue makes the average stiffness higher than in the calf for example. If the identification had been made over the whole leg, the resulting identified 
material parameters would have been an average between the stiffness at the different heights.

Besides, the three-dimensional model also accounts for the out-of-plane displacements and assures a realistic restitution of the leg response in the region where the cost function is applied. This constitutes an added value of the present model over the previous 2D model in which plane strain assumption was made [14].

\subsection{Comfort}

Comfort is a very important issue in the treatment of vein-related diseases by MCS. Indeed, if the patient cannot endure the treatment prescribed by the doctor because of pain issues, he/she will be inclined to neglect the treatment even if it has a positive impact.

Although the issue of comfort is not very well understood, recent studies on the underlying mechanical principles of discomfort have shown that mechanical loads (pressure as well as shear) influences discomfort [15] [16]. In particular, the Pain Pressure Threshold (PPT) is an index widely used to evaluate the perceived pain caused by high local pressure [17][18][19].

Our simulation results show that the maximum hydrostatic pressure in the soft tissues is related to the maximum contact pressure on the skin. In addition, according to Laplace law, the contact pressure is inversely proportional to the curvature radius for a given sock tension. We can therefore deduce from Laplace law that in a round leg there is no concentration of interface pressure on the skin. Besides, a high proportion of fat always results in a round leg. It is therefore sound to suppose that the morphology of the fat tissue can be used to estimate the comfort of the MCS. This statement however deserves further investigation.

\section{CONCLUSIONS}

In order to provide a quantitative insight into the mechanical response of soft tissues of the leg to elastic compression and to address the essential question of the transmission of pressure, three-dimensional patient-specific 
FE models of the leg was developed for six subjects. In order to identify the non-linear properties of the soft tissues, an inverse method based on Finite Element Model Updating has been presented. This constitutes the original contribution of this work.

The material properties identified for the superficial and deep soft tissues are in good agreement with the values reported in the literature for fat and muscles.

Our results show that the mechanical response of the leg to external compression results in a non-homogeneous pressure field which supports the relevance of a patient-specific treatment of the evaluation of the performance of MCS. It has also been shown that the mean interface pressure applied by the MCS can be used as an indicator of the efficiency of the MCS and that, the thickness of the adipose tissue in the leg can be used to estimate the comfort of the MCS.

Yet, the relationship between the hydrostatic pressures measured in the vicinity of the veins using the developed model and the impact on veinrelated diseases needs to be further investigated to improve our current understanding of the mechanisms of action of MCS. To address the latter point, a more realistic model will be implemented in $2 \mathrm{D}$ to analyse the local response of the vein wall. Refinement of the muscle models are also under progress.

\section{References}

[1] M. R. Nehler, G. L. Moneta, D. M. Woodard, R. D. Defrang, C. T. Harker, L. M. Taylor Jr., and J. M. Porter, "Perimalleolar subcutaneous tissue pressure effects of elastic compression stockings," Journal of Vascular Surgery, vol. 18, no. 5, pp. 783-788, Nov. 1993.

[2] V. Ibegbuna, K. T. Delis, A. N. Nicolaides, and O. Aina, "Effect of elastic compression stockings on venous hemodynamics during walking," Journal of Vascular Surgery, vol. 37, no. 2, pp. 420-425, Feb. 2003.

[3] J. C. Mayberry, G. L. Moneta, R. D. De Frang, and J. M. Porter, "The influence of elastic compression stockings on deep venous hemodynamics," Journal of Vascular Surgery, vol. 13, no. 1, pp. 91-100, Jan. 1991.

[4] C. M. Hamel-Desnos, B. J. Guias, P. R. Desnos, and A. Mesgard, "Foam Sclerotherapy of the Saphenous Veins: Randomised Controlled Trial with or without Compression," Eu- 
ropean Journal of Vascular and Endovascular Surgery, vol. 39, no. 4, pp. 500-507, Apr. 2010.

[5] P. Kern, A.-A. Ramelet, R. Wütschert, and D. Hayoz, "Compression after sclerotherapy for telangiectasias and reticular leg veins: A randomized controlled study," Journal of Vascular Surgery, vol. 45, no. 6, pp. 1212-1216, Jun. 2007.

[6] C. J. Wildin, A. C. W. Hui, C. N. A. Esler, and P. J. Gregg, "In vivo pressure profiles of thigh-length graduated compression stockings," British Journal of Surgery, vol. 85, no. 9, pp. 1228-1231, Sep. 1998.

[7] I. Gaied, S. Drapier, and B. Lun, "Experimental assessment and analytical 2D predictions of the stocking pressures induced on a model leg by Medical Compressive Stockings," Journal of Biomechanics, vol. 39, no. 16, pp. 3017-3025, 2006.

[8] X. Dai, R. Liu, Y. Li, M. Zhang, and Y. Kwok, “Computational Textile," vol. 55, Springer Berlin / Heidelberg, 2007, pp. 301-309.

[9] S. Avril, P. Badel, L. Dubuis, P.-Y. Rohan, J. Debayle, S. Couzan, and J.-F. Pouget, "Patient-Specific Modeling of Leg Compression in the Treatment of Venous Deficiency," in Patient-Specific Modeling in Tomorrow's Medicine, vol. 09, A. Gefen, Ed. Berlin, Heidelberg: Springer Berlin Heidelberg, 2011, pp. 217-238.

[10] L. Dubuis, S. Avril, J. Debayle, and P. Badel, "Identification of the material parameters of soft tissues in the compressed leg," Computer Methods in Biomechanics and Biomedical Engineering, vol. 15, no. 1, pp. 3-11, 2012.

[11] R. Liu, Y.-L. Kwok, Y. Li, T.-T. Lao, X. Zhang, and X. Dai, “A three-dimensional biomechanical model for numerical simulation of dynamic pressure functional performances of graduated compression stocking (GCS)," Fibers and Polymers, vol. 7, no. 4, pp. 389397, Dec. 2006.

[12] T. J. Carter, M. Sermesant, D. M. Cash, D. C. Barratt, C. Tanner, and D. J. Hawkes, "Application of soft tissue modelling to image-guided surgery," Medical Engineering \& Physics, vol. 27, no. 10, pp. 893-909, Dec. 2005.

[13] M. Kauer, V. Vuskovic, J. Dual, G. Szekely, and M. Bajka, "Inverse finite element characterization of soft tissues," Medical Image Analysis, vol. 6, no. 3, pp. 275-287, Sep. 2002.

[14] S. Avril, L. Bouten, L. Dubuis, S. Drapier, and J.-F. Pouget, "Mixed Experimental and Numerical Approach for Characterizing the Biomechanical Response of the Human Leg Under Elastic Compression," Journal of Biomechanical Engineering, vol. 132, no. 3, pp. 31006-31014, 2010.

[15] R. H. M. Goossens, "Fundamentals of Pressure, Shear and Friction and Their Effects on the Human Body at Supported Postures," in Bioengineering Research of Chronic Wounds, vol. 1, A. Gefen, Ed. Springer Berlin Heidelberg, 2009, pp. 1-30.

[16] J. C. Moreno, F. J. Brunetti, J. L. Pons, J. M. Baydal, and R. Barbera, "Rationale for Multiple Compensation of Muscle Weakness Walking with a Wearable Robotic Orthosis," in Robotics and Automation, 2005. ICRA 2005. Proceedings of the 2005 IEEE International Conference on, 2005, pp. $1914-1919$.

[17] A. A. Fischer, "Pressure tolerance over muscles and bones in normal subjects," Arch Phys Med Rehabil, vol. 67, no. 6, pp. 406-409, Jun. 1986.

[18] A. A. Fischer, "Pressure algometry over normal muscles. Standard values, validity and reproducibility of pressure threshold," Pain, vol. 30, no. 1, pp. 115-126, Jul. 1987.

[19] J. Ylinen, E.-P. Takala, H. Kautiainen, M. Nykänen, A. Häkkinen, T. Pohjolainen, S.-L. Karppi, and O. Airaksinen, "Effect of long-term neck muscle training on pressure pain threshold: A randomized controlled trial," European Journal of Pain, vol. 9, no. 6, pp. 673-681, Dec. 2005. 